\title{
Concentration of fetal plasma and amniotic fluid interleukin-1 in pregnancies complicated by preterm prelabour amniorrhexis
}

\author{
S G Carroll, A Abbas, Y Ville, N Meher-Homji, K H Nicolaides
}

\begin{abstract}
Aims-To determine interleukin-1及 (IL-

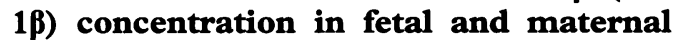
plasma and amniotic fluid from pregnancies complicated by preterm prelabour amniorrhexis and to define the relation of this cytokine to intrauterine infection and the onset of labour.
\end{abstract}

Methods-Cross-sectional study of 23 pregnancies complicated by preterm prelabour amniorrhexis. Enzyme linked im-

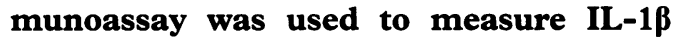
concentration in fetal and maternal plasma and amniotic fluid. In each case, fetal blood and amniotic fluid were cultured for micro-organisms.

Results-In pregnancies with positive fetal blood and/or amniotic fluid cultures, plasma and amniotic fluid concentrations of IL-1B were higher and the interval between amniorrhexis and onset of labour was shorter than in the non-infected group. There were no significant associations between fetal plasma IL-1 $\beta$ and

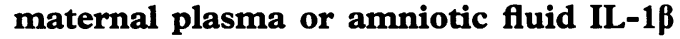
concentrations, fetal leucocyte count or the interval between amniorrhexis and the onset of labour.

Conclusions-These findings suggest that although intrauterine infection is associated with increased IL-1B concentrations in fetal plasma and amniotic fluid, there is no significant association between the concentration of IL-1B and the interval between amniorrhexis and the onset of labour.

(f Clin Pathol 1995;48:368-371)

Keywords: Interleukin-1, cordocentesis, amniocentesis, preterm prelabour amniorrhexis.

Harris Birthright Research Centre for Fetal Medicine, King's College Hospital Medical School,

Denmark Hill,

London SE5 8RX

$S$ G Carroll

A Abbas

$\mathrm{Y}$ Ville

N Meher-Homj

$\mathrm{K} \mathrm{H}$ Nicolaides

Correspondence to: Dr K H Nicolaides.

Accepted for publication: 25 August 1994
Interleukin-1 $\beta$ (IL-1 $\beta$ ), which is produced by monocytes, macrophages, lymphocytes, and epithelial cells, has been implicated in the pathogenesis of preterm labour. Romero et $a l^{1}$ reported that the concentration of $\mathrm{IL}-1 \beta$ in amniotic fluid was higher in pregnancies complicated by both microbial invasion of the amniotic cavity and preterm labour than in pregnancies with either preterm labour or microbial invasion of the amniotic cavity alone. In vitro studies have shown that both IL-1 $\beta$ and bacterial products stimulate prostaglandin release from the human amnion, ${ }^{23}$ and it was postulated that in the presence of infection there is release of cytokines which stimulate amniotic membranes to synthesise prostaglandins that induce uterine contractions. ${ }^{145}$

The aim of this study was to determine whether, in pregnancies with preterm prelabour amniorrhexis, the concentration of IL- $1 \beta$ in fetal blood and amniotic fluid is related to the presence of intrauterine infection and to the interval between membrane rupture and the onset of labour.

\section{Methods}

IL- $1 \beta$ was measured in fetal blood from 23 pregnancies complicated by preterm prelabour amniorrhexis at 21-37 weeks of gestation (mean 28 weeks) and the values were compared with those of 75 controls. The women with amniorrhexis were referred to our centre for amniocentesis and cordocentesis within three days of membrane rupture to establish the presence of intrauterine infection. The patients gave written informed consent to participate in the study which was approved by the Hospital Ethics Committee.

The diagnosis of amniorrhexis was confirmed by the ultrasonographic demonstration of decreased or absent amniotic fluid and the visualisation of nitrazine positive fluid in the vagina. Cordocentesis and amniocentesis were performed using a single uterine transabdominal entry of a $20 \mathrm{G}$ needle under ultrasound guidance. In all cases umbilical venous blood was obtained and the Kleihauer-Betke test confirmed that all blood samples contained only fetal blood.

Fetal and maternal blood (obtained from the antecubital vein just before cordocentesis) were inoculated into aerobic and anaerobic blood culture bottles (Bactec, Becton-Dickinson, Sparks, Maryland, USA). The amniotic fluid was cultured using standard microbiological techniques and also inoculated into Mycofast liquid cultures for Ureaplasma urealyticum (U.u.) and Mycoplasma hominis (M.h.: International Mycoplasma S.A., Toulon, France).

The patients were divided into three groups depending on the results of cultures. Group 1 included those patients with negative cultures of amniotic fluid and fetal blood. Group 2 consisted of patients who had positive amniotic fluid but negative fetal blood cultures. In group 3 the patients had positive fetal blood cultures.

In 18 cases the pregnancies were managed expectantly and the onset of labour was spontaneous. There were four inductions of labour, two each from groups 2 and 3 and one termination of pregnancy from group 3 . The 
placentae were examined histologically for evidence of chorioamnionitis, which was defined by a polymorphonuclear leucocytic infiltration of the extraplacental membranes, the chorionic plate or umbilical cord blood vessels.

In the control group fetal blood samples were obtained either by fetal cardiocentesis from women undergoing elective terminations of pregnancy for social indications at 12-17 weeks of gestation $(n=22)$, by cordocentesis at $18-37$ weeks from women undergoing prenatal diagnosis $(n=39)$ or by umbilical cord puncture at elective caesarean section at term for either breech presentation or previous caesarean section $(n=14)$. The indications for cordocentesis were fetal karyotyping because of advanced maternal age $(n=4)$, assessment of red blood cell isoimmunised pregnancies $(n=3)$ and karyotyping for fetal malformations, such as hydronephrosis, facial cleft or choroid plexus cysts $(n=32)$. In all cases the fetal abdominal circumference, haemoglobin concentration and leucocyte count were within the appropriate reference range for gestation and the fetal karyotype was normal. In all red blood cell isoimmunised pregnancies included in this study the fetuses were Coomb's negative.

Fetal blood samples $(200 \mu \mathrm{l})$ were used for full blood count (Coulter S-Plus counter, Coulter Electronics, Luton, UK) and blood films to determine the leucocyte count. Fetal blood samples $(400 \mu \mathrm{l})$ were also collected into $20 \mu \mathrm{l}$ of isotonic edetic acid solution $(0.5 \mathrm{mmol} / 1$ in $0.15 \mathrm{mmol} / \mathrm{l}$ sodium chloride) and centrifuged immediately at $0-4^{\circ} \mathrm{C}$ to avoid contamination with endotoxin and in vitro production of cytokine. ${ }^{6}$ The separated plasma was stored at $-20^{\circ} \mathrm{C}$. The plasma IL-1 $\beta$ concentration was measured by enzyme linked immunoassay (Medgenix Diagnostics, Brussels, Belgium), which can detect minimal concentrations of $0.3 \mathrm{IU} / \mathrm{ml}$. The intra-and interassay coefficients of variation were $3.4 \%$ and $4.4 \%$, respectively. In the amniorrhexis group the IL-1 $\beta$ concentration was also measured in maternal blood and amniotic fluid obtained at the time of amniocentesis and cordocentesis.

Regression analysis was used to determine whether the fetal blood IL-1 $\beta$ concentration in

Median (range) for fetal plasma, amniotic fluid and maternal plasma IL-1 $\beta$ concentrations, fetal and maternal leucocyte count and gestational age at preterm prelabour amniorrhexis (group 1, no infection; group 2, microbial invasion of the amniotic cavity; group 3, fetal bacteraemia). The time interval between amniorrhexis and delivery in the 18 pregnancies with spontaneous labour (eight in group 1, four in group 2, and six in group 3) was also recorded

\begin{tabular}{|c|c|c|c|}
\hline & $\begin{array}{l}\text { Group 1 } \\
(n=10)\end{array}$ & $\begin{array}{l}\text { Group } 2 \\
(n=6)\end{array}$ & $\begin{array}{l}\text { Group } 3 \\
(n=7)\end{array}$ \\
\hline $\begin{array}{l}\text { Fetal plasma } \\
\text { IL-1 (IU/ml) } \\
\text { Amniotic fluid } \\
\text { IL-1 } \beta \text { (IU/ml) } \\
\text { Maternal plasma } \\
\text { IL-1 } \beta \text { (IU/ml) } \\
\text { Fetal leucocyte count } \\
\left(10^{9} / 1\right) \\
\text { Maternal leucocyte count } \\
\left(10^{9} / 1\right) \\
\text { Gestation at } \\
\text { amniorrhexis (weeks) } \\
\text { Amniorrhexis to delivery } \\
\text { interval (days) }\end{array}$ & $\begin{array}{l}11 \cdot 5 \\
(0 \cdot 1-37 \cdot 3) \\
18 \cdot 8 \\
(0 \cdot 4-566 \cdot 7) \\
1 \cdot 5 \\
(0 \cdot 1-51 \cdot 4) \\
5 \cdot 5 \\
(3 \cdot 5-10 \cdot 5) \\
9 \cdot 5 \\
(6 \cdot 3-16 \cdot 0) \\
32 \\
(22-37) \\
19 \\
(1-45)\end{array}$ & $\begin{array}{l}74 \cdot 2^{*} \\
(0 \cdot 1-254 \cdot 9) \\
32 \cdot 8 \\
(0 \cdot 1-1544 \cdot 8) \\
7 \cdot 9 \\
(0 \cdot 1-179 \cdot 3) \\
8 \cdot 5 \\
(3 \cdot 3-13 \cdot 5) \\
9 \cdot 1 \\
(5 \cdot 8-17 \cdot 4) \\
28 \\
(22-33) \\
17 \\
(1-22)\end{array}$ & $\begin{array}{l}286 \cdot 5^{* *} \\
(83 \cdot 1-398 \cdot 2) \\
800^{*} \\
(7 \cdot 4-4504 \cdot 4) \\
2 \cdot 6 \\
(0 \cdot 1-11 \cdot 0) \\
6 \cdot 4 \\
(1 \cdot 5-9 \cdot 1) \\
13 \cdot 5 \\
(7 \cdot 1-22 \cdot 9) \\
26 \\
(21-34) \\
2 * * \\
(1-5)\end{array}$ \\
\hline
\end{tabular}

${ }^{*} p<0.05,{ }^{* *} p<0.01$. the control group was significantly associated with gestational age and leucocyte count. The rank analysis of variance test was used to examine the significance of differences in fetal plasma and amniotic fluid IL- $1 \beta$ concentrations between the amniorrhexis subgroups. In the amniorrhexis group regression analysis was used to determine the significance of associations between fetal blood and amniotic fluid IL- $1 \beta$ concentrations and the interval between membrane rupture and delivery in the 18 patients with spontaneous onset of labour.

\section{Results}

In the control group the gestation at fetal blood sampling was $12-38$ (mean 24 ) weeks. There were no significant associations between the plasma IL-1 $\beta$ concentration and gestation $(\mathrm{r}=0.01$, median $21.63 \mathrm{IU} / \mathrm{ml}$, range $0.01-$ $48 \cdot 16 \mathrm{IU} / \mathrm{ml}$ ) or fetal blood leucocyte count $(r=-0.036)$.

Of the patients with amniorrhexis 10 were in group 1 , six in group 2 (five with positive amniotic fluid cultures of M.h. and/or U.u. and one with Streptococcus agalactiae) and seven in group 3 (one case each with positive fetal blood cultures of Lactobacillus spp, Enterobacter spp, Streptococcus agalactiae, Streptococcus viridans, Streptococcus milleri, Citrobacter spp, and Candida albicans).

In groups 2 and 3 fetal plasma and amniotic fluid IL-1 $\beta$ concentrations were significantly higher than values in group 1 and controls (table, figure). Postpartum histological evidence for chorioamnionitis was present in all 13 cases in groups 2 and 3 and in one of the 10 cases in group 1 . IL-1 $\beta$ concentrations were significantly higher in both fetal plasma $(z=2.97, p<0.01)$ and amniotic fluid $(z=2.01, p<0.05)$ in the cases with than in those without chorioamnionitis. The fetal plasma IL-1 $\beta$ concentration was not significantly associated with amniotic fluid IL-1 $\beta(r=0.348)$ or maternal plasma IL-1 $\beta(r=-0 \cdot 139)$ concentrations, or the fetal leucocyte count $(r=-0 \cdot 355)$.

In the 18 patients who underwent spontaneous labour, group 3 patients had a significantly shorter amniorrhexis to delivery interval than those in groups 1 and 2 . However, there were no significant associations between fetal plasma or amniotic fluid IL-1 $\beta$ concentrations and amniorrhexis to delivery interval $(n=18, r=-0.423$ and $r=-0.393$, respectively).

\section{Discussion}

The results of this study demonstrate that in normal pregnancy IL- $1 \beta$ is present in the fetal circulation from at least 12 weeks of gestation and the plasma concentrations are not related to the leucocyte count or the gestational age. In pregnancies with amniorrhexis and intrauterine infection fetal blood and amniotic fluid IL-1 $\beta$ concentrations are increased and the interval between amniorrhexis and delivery is reduced. However, there is no significant association between IL-1 $\beta$ concentrations and interval to delivery. 

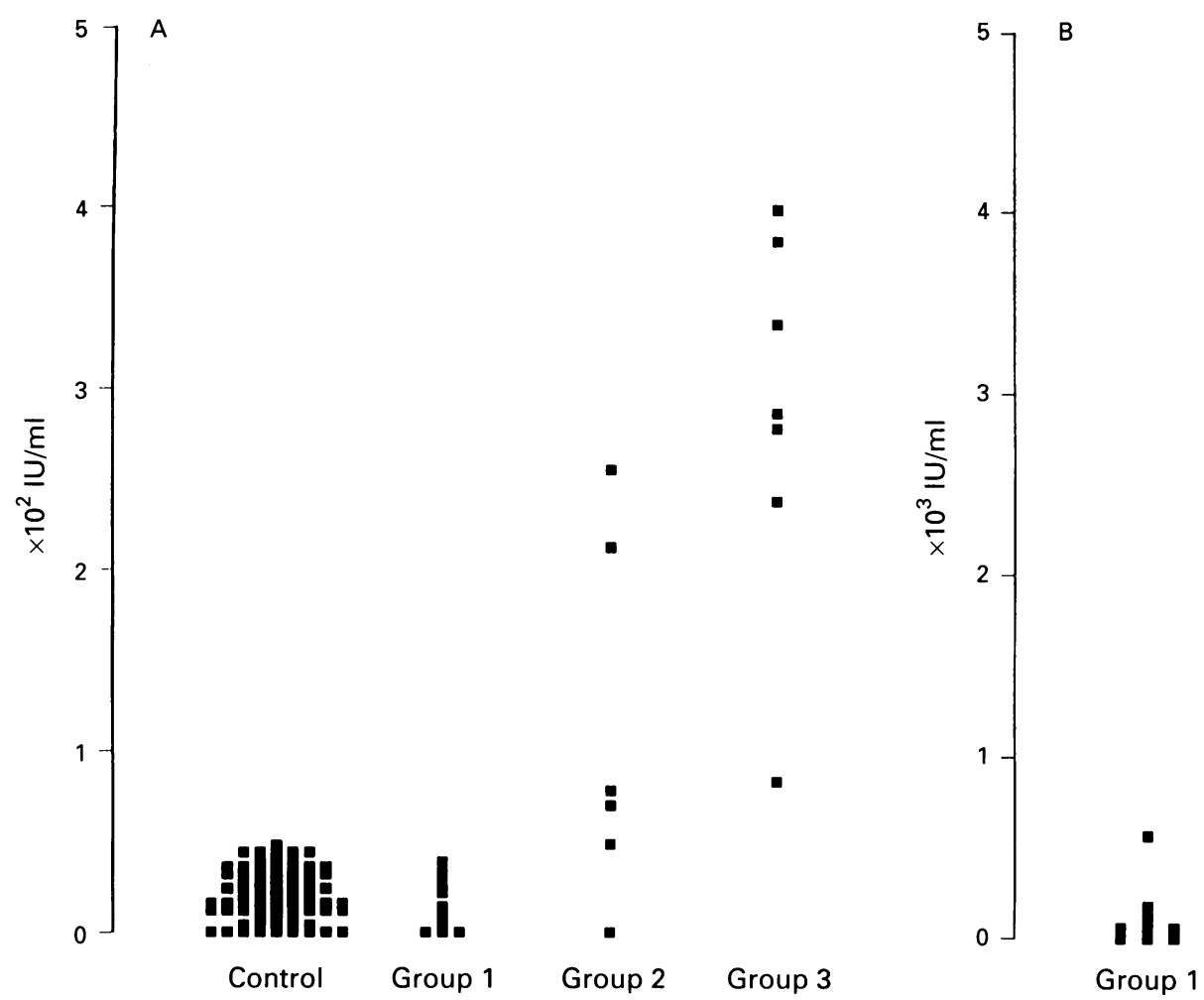

Fetal plasma (A) and amniotic fluid (B) IL-1 $\beta$ concentrations in controls and in pregnancies complicated by preterm prelabour amniorrhexis (group 1 , no infection; group 2, microbial invasion of the amniotic cavity; group 3, fetal bacteraemia).

IL- $1 \beta$ concentrations in plasma and amniotic fluid do not necessarily accurately reflect the amount produced or released into these compartments. Binding proteins such as IgG autoantibodies, $\alpha_{2}$-macroglobulin and soluble receptors shed from cells following inflammatory episodes can influence the half-life of cytokines in the circulation and their distribution in other extracellular compartments. ${ }^{6-9}$ Furthermore, cytokines usually act in synergy with or in opposition to other cytokines. In addition, a more complete picture of the biological role of IL- $1 \beta$ in the infectious process necessitates measurement of IL- $1 \beta$ receptor antagonists as well as of the agonist. ${ }^{6}$ Nevertheless, the main aim of this study was to compare circulating concentrations of IL- $1 \beta$ in normal and a group of pathological pregnancies rather than to quantify the production and biological activity of this cytokine.

In this study the diagnosis of intrauterine infection was based on the results of fetal blood and amniotic fluid cultures. Many authors have advocated the use of amniocentesis for the diagnosis of intrauterine infection in cases of preterm prelabour amniorrhexis because the clinical signs of infection, such as maternal pyrexia or leucocytosis, are not specific and they develop late in the course of the disease. ${ }^{1011}$ However, in postnatal studies, the vast majority of infants with positive cultures of skin swabs or gastric aspirates (the equivalent of positive amniotic fluid cultures) are not infected and do not suffer any morbidity, unlike those who are shown to have bacteraemia. ${ }^{12}$ We assumed that the same may be true for the fetus, hence our protocol for the management of amniorrhexis includes culture of fetal blood.
There was an association between infection and increased fetal plasma and amniotic fluid IL- $1 \beta$ concentrations. This is consistent with previous reports of increased concentrations of amniotic fluid IL-1 $\beta$, other cytokines and prostaglandins in cases of positive amniotic fluid cultures and preterm labour. ${ }^{1314}$ Chorioamnionitis may cause release of IL- $1 \beta$ into both the fetal circulation and the amniotic fluid. In vitro studies have demonstrated release of IL$1 \beta$ by decidual and placental macrophages and syncytiotrophoblasts. ${ }^{1516}$

The lack of a significant association between fetal plasma and amniotic fluid IL-1 $\beta$ concentrations does not preclude a common source of IL-1 $\beta$; findings in a cross-sectional study do not allow conclusions to be drawn on the dynamic inter-relation between two biological compartments. As the maternal plasma IL-1 $\beta$ concentration was not increased in the infected group, it is unlikely that transplacental transfer from the mother can explain the findings in fetal plasma and amniotic fluid. Although there was no significant association between fetal leucocyte counts and fetal plasma IL-1 $\beta$ concentrations, this does not exclude fetal leucocytes as the source of IL-1 $\beta$; in a previous study we demonstrated that fetal infection is associated with changes in lymphocyte subpopulations in the presence of normal leucocyte counts. ${ }^{17}$

The association between infection and a shorter latency interval is compatible with the findings of previous studies, that in preterm prelabour amniorrhexis patients with chorioamnionitis deliver earlier than those without infection. ${ }^{1819}$ The lack of a significant association between fetal plasma or amniotic fluid 
IL-1 $\beta$ concentrations and the interval between amniorrhexis and onset of labour is in apparent contradiction with the hypothesis that there is a direct causal association between infection, cytokines, prostaglandins, and labour. It is acknowledged, however, that the lack of a statistical association does not rule out a role for cytokines in the initiation of labour. Gravett et $a l^{20}$ have demonstrated that in chronically instrumented pregnant rhesus monkeys intra-amniotic inoculation of Streptococcus agalactiae was associated with an increase in IL-1 $\beta$ and prostaglandin concentrations after 18 hours of inoculation and 10 hours before the onset of uterine contractions. As the relation between infection, cytokines and labour is a temporal one, it is not surprising that in our cross-sectional study it was not possible to demonstrate a significant association between the IL-1 $\beta$ concentration and the latency interval.

1 Romero R, Brody DT, Oyarzun E, Mazor M, King Wu Y, Hobbins JC, et al. Interleukin-1: A signal for the onset of parturition. Am 7 Obstet Gynecol 1989;160:117-23.

2 Lamont RF, Rose MP, Elder MG. Effect of bacterial products on prostaglandin $\mathrm{E}$ production by amnion cells. Lancet 1985;ii:331-3

3 Romero R, Durum S, Dinarello CA, Oyarzun E, Hobbins JC, Mitchell MD. Interleukin-1 stimulates prostaglandin biosynthesis by the human amnion. Prostaglandins 1989;37: 13-22.

4 Novy JM, Liggins GC. Role of prostaglandins, prostacyclin and thromboxanes in the physiologic control of the uterus and in parturition. Semin Perinatol 1980;4:5-66.

5 Mitchell MD. The mechanisms of human parturition. $f$ Dev Physiol 1984;6:107-18.

6 Cannon JG, Nerad JL, Poutsiaka DD, Dinarello CA. Measuring circulating cytokines. $\mathcal{F}$ App Physiol 1993;75:1897902 .

7 Matsuda T, Hirano T, Nagasawa S, Kishioto T. Identification of a2-macroglobulin as a carrier protein for IL-6. 7 Immunol 1989;142:148-52.

8 Novick D, Engelmann H, Wallach D, Rubinstein M. Soluble cytokine receptors are present in normal human urine. $f$ Exp Med 1989;170:1409-14

9 Engelmann H, Novick D, Wallach D. Two tumor necrosis factor binding proteins purified from human urine. Evidence for immunological cross-reactivity with cell surface tumor necrosis factor receptors. F Biol Chem 1990;265: 1531-6.

10 Bobitt JR, Ledger WJ. Amniotic fluid analysis. Its role in maternal and neonatal infection. Obstet Gynecol 1978;51: $57-67$.

11 Kappy KA, Cetrulo CL, Knuppel RA, Ingardia CJ, Sbarra AJ, Scerbo JC, et al. Premature rupture of the membranes: A conservative approach. Am $\mathcal{f}$ Obstet Gynecol 1979;134: 655-61.

12 Thompson PJ, Greenough A, Gamsu H, Nicolaides K, Philpott-Howard J. Congenital bacterial sepsis in very preterm infants. $\mathcal{F}$ Med Microbiol 1991;36:1-4.

13 Potter NT, Kosuda L, Bigazzi PE, Fleming AD, Vintzileos $\mathrm{AM}$, Homon C, et al. Relationships among cytokines (IL1, TNF and IL-8) and histologic markers of acute ascending intrauterine infection. Fournal of Maternal-Fetal Medicine 1992;1:142-7.

14 Hillier SL, Witkin SS, Krohn MA, Watts DH, Kiviat NB, Eschenbach DA. The relationship of amniotic fluid cytokines and preterm delivery, amniotic fluid infection, histologic choriomnionitis, and chorioamnion infection. Obstet Gynecol 1993;81:941-8.

15 Romero R, King Wu Y, Brody DT, Oyarzun E, Duff GW, Durum SK. Human decidua: A source of Interleukin-1. Obstet Gynecol 1989;73:31-4.

16 Taniguchi T, Matsukaki N, Kameda T, Shimoya K, Toushun J, Saji F, et al. The enhanced production of placental interleukin-1 during labor and intrauterine infection. $A m \mathcal{F}$ Obstet Gynecol 1991;165:131-7.

17 Thilaganathan B, Carroll SG, Plachouras N, Makrydimas G, Nicolaides KH. Fetal immunological and haematological changes in intrauterine infection. Br fObstet Gynaecol 1994; 101:418-21.

18 Schreiber J, Benedetti T. Conservative management of preterm premature rupture of the fetal membranes in a low socioeconomic population. Am f Obstet Gynecol 1980;136: 92-6.

19 Moretti M, Sibai BM. Maternal and perinatal outcome of expectant management of premature rupture of membranes in the midtrimester. Am $\mathcal{F}$ Obstet Gynecol 1988;159: $390-6$

20 Gravett MG, Witkin SS, Haluska GJ, Edwards JL, Cook MJ, Novy MJ. An experimental model for intraamniotic infection and preterm labor in rhesus monkeys. Am f Obstet Gynecol 1994;171:1660-7. 